\title{
PROPERTIES OF SIMPLE AND GENERALIZED LAGUERRE FUNCTIONS FOR TIME-DELAY SYSTEM APPROXIMATIONS
}

\section{Norbert Zsitva}

Department of Control and Instrumentation, Brno University of Technology, Czech Republic xzsitv00@stud.feec.vutbr.cz

\begin{abstract}
The properties of the simple and generalized Laguerre functions for time-invariant system approximations are discussed. The expressions for these functions are presented and the differences between them are shown. The approximations heavily depend on the free parameters of the Laguerre functions. Because of this, the optimal choice of these parameters is described for both the simple and the generalized functions. This assures that the results are satisfactory. The approximations are shown on two different systems. These approximations are evaluated with the help of the quadratic error criterion. It is shown that the results differ for the two chosen systems. The reason behind this is explained with the help of the Laguerre functions' properties and the initial value theorem.
\end{abstract}

Keywords: Laguerre functions, time-delay system, system approximation, optimal free parameters, impulse response, transfer function.

\section{Introduction}

This paper deals with the approximation of systems with orthogonal functions. This topic appears in numerous literature. Similarly, there are many research papers which mention the simple Laguerre functions (SLF). However, this is not the case for the generalized Laguerre functions (GLF), which appear less frequently when discussing approximations.

In paper [1] it was described that the generalized Laguerre functions give better results for most system approximations. The exceptions were the low order of the approximation and the small value of the time delay. The aim of this paper is to further expand these results. This is achieved by describing the properties of the Laguerre functions and by showing which functions give better results depending on the systems' characteristics. This is done with the help of two chosen systems for which the approximations are shown for different values of time delay. It is stated which system properties are important for the generalized Laguerre functions to give better results.

\section{Generalized and Simple Laguerre Functions}

The GLFs are defined by equation [2]:

$$
\lambda_{m}^{(\alpha)}(\sigma ; t)=\sqrt{\frac{\sigma m !}{\Gamma(m+\alpha+1)}} \sum_{n=0}^{m}(-1)^{n}\left(\begin{array}{c}
m+\alpha \\
m-n
\end{array}\right) \frac{(\sigma t)^{n+\frac{\alpha}{2}}}{n !} e^{-\sigma \frac{t}{2}}
$$

$m$ is the function's order $(m \geq 0), t$ represents the time $(t>0)$ and $\Gamma(m+\alpha+1)$ is the gamma function. These functions have two free parameters. Parameter $\alpha$ is the order of generalization $(\alpha>-1)$ and $\sigma$ is the timescale. If $\alpha=0$ is substituted to equation (1), the SLFs are obtained, which will be represented by $\lambda_{m}(\sigma ; t)$ :

$$
\lambda_{m}(\sigma ; t)=\sqrt{\sigma} \sum_{n=0}^{m}(-1)^{n}\left(\begin{array}{c}
m \\
m-n
\end{array}\right) \frac{(\sigma t)^{n}}{n !} e^{-\sigma \frac{t}{2}} .
$$

The Laguerre functions form a complete orthonormal set in $L_{2}\left(\mathbb{R}^{+}\right)[2]$. Due to this, any causal function $f(t) \in L^{2}$ can be expressed with it's Laguerre series according to [2, 3]:

$$
f(t)=\sum_{m=0}^{\infty} C_{m} \lambda_{m}^{(\alpha)}(\sigma ; t)
$$

Coefficient $C_{m}$ is expressed by the inner product of functions $f(t)$ and $\lambda_{m}^{(\alpha)}(\sigma ; t)$ : 


$$
C_{m}=\left\langle f(t), \lambda_{m}^{(\alpha)}(\sigma ; t)\right\rangle
$$

For numerical computation equation (3) will be used as

$$
f(t)=\sum_{m=0}^{M} C_{m} \lambda_{m}^{(\alpha)}(\sigma ; t),
$$

where the upper limit of the sum was changed to $M$, which represents the order of the approximation.

\section{Differences Between the Simple and Generalized Laguerre Functions}

As mentioned above in section 2, the difference between the GLFs and SLFs lies in the free parameter $\alpha$. As already stated in [1], the GLFs give better approximations than the SLFs for systems with higher values of timedelay. The reason for this is the order of generalization $\alpha$. The main influence of this parameter is shifting the GLFs to the right on the time axis. For time-delay system approximations this property is especially important because this behaviour is similar to the increase of the time delay in impulse responses.

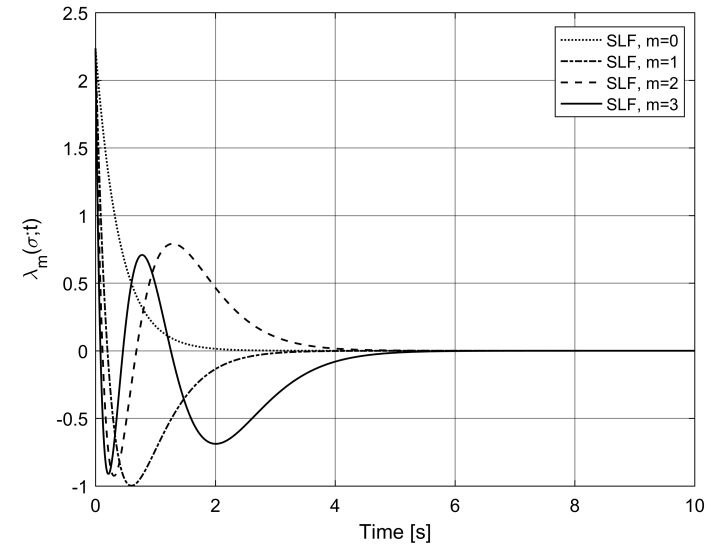

(a) Simple Laguerre functions for $\sigma=5$

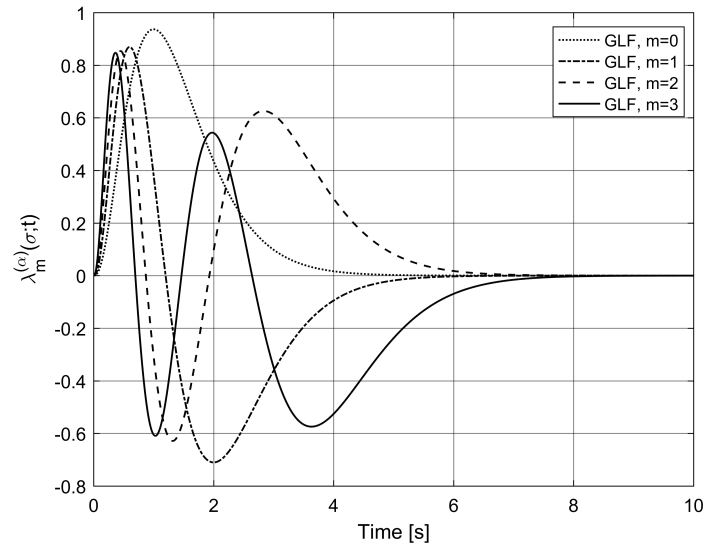

(b) Generalized Laguerre functions for $\sigma=5, \alpha=5$

Figure 1: The first four simple and generalized Laguerre functions

\subsection{The Initial Point of the Laguerre Functions}

The simple and generalized Laguerre functions are displayed in Fig. 1. Fig. 1b clearly shows that the GLFs' value is zero for $t=0$. This can also be proven analytically. After substituting $t=0$ into equation (1), the expression $(\sigma t)^{n+\frac{\alpha}{2}}$ becomes zero for all $n$ values. This means that the generalized Laguerre functions' value for $t=0$ is also zero

$$
\lambda_{m}^{(\alpha)}(\sigma ; 0)=0
$$

In the case of the SLFs, the behaviour for $t=0$ is somewhat different. This is because the expression $(\sigma t)^{n}$ for different $n$ values is as follows:

$$
(\sigma t)^{n}= \begin{cases}1, & n=0 \\ 0, & n \neq 0, t=0\end{cases}
$$

This means that for the SLFs the value of the summation is zero if $n>0$. However, if $n=0$ the summation's value is 1 for $t=0$. This way for the SLFs we obtain

$$
\lambda_{m}(\sigma ; 0)=\sqrt{\sigma}
$$

This result corresponds with Fig. 1a, where $\sigma=5$, and the SLFs' value for $t=0$ is $\sqrt{5}$. 


\section{Optimal Values of the Free Parameters}

The choice of the values of the free parameters for both the SLFs and GLFs is described in [1]. For the GLFs the following equations are used [4]:

$$
\begin{gathered}
\alpha=\frac{2 m_{0}}{m_{-1}} \sqrt{\frac{m_{-1} \mu_{1}}{m_{-1} m_{1}-m_{0}^{2}}}, \\
\sigma=2 \sqrt{\frac{m_{-1} \mu_{1}}{m_{-1} m_{1}-m_{0}^{2}}} .
\end{gathered}
$$

For the SLFs the result

$$
\sigma=2 \sqrt{\frac{\mu_{1}}{m_{1}}}
$$

is obtained from [5] as explained in [1]. The variables $m_{i}$ and $\mu_{i}$ are "moments" or signal measurements. They are defined as [4]:

$$
\begin{array}{ll}
m_{i}=\left\langle f(t), g_{i}(t)\right\rangle, & g_{i}(t)=t^{i} f(t), \\
\mu_{i}=\left\langle f^{\prime}(t), \tilde{g}_{i}(t)\right\rangle, & \tilde{g}_{i}(t)=t^{i} f^{\prime}(t) .
\end{array}
$$

Using equations (9), (10) and (11) ensures that the approximations give optimal results.

\section{Evaluation of the Approximations}

For the evaluation of the results, the quadratic criterion will be used as defined in [5]. As already stated in [1], this expression can be modified, so it only contains the coefficient $C_{m}$ :

$$
J=1-\frac{\sum_{m=0}^{M} C_{m}^{2}}{\sum_{m=0}^{\infty} C_{m}^{2}} .
$$

The denominator of the fraction in (14) equals to the signal measurement $m_{0}$ because both of these expressions represent signal energy. This way equation (14) can be rewritten as

$$
J=1-\frac{\sum_{m=0}^{M} C_{m}^{2}}{m_{0}} .
$$

The main advantage of this lies in simplifying the numerical computations. Coefficient $C_{m}$ is already calculated for every $m$ for the Laguerre functions, $m_{0}$ is also known at this point because it is needed to obtain the free parameters.

\section{System Approximation With Time Delay}

The GLFs and SLFs will be used to approximate the impulse response of linear time-invariant systems with time-delay with the help of expression (5) from section 2. The free parameters are computed numerically according to equations (9), (10) and (11). For simple systems, these parameters can be calculated analytically. This results in higher precision and faster computation time.

The approximations will be shown on two time-delay systems with transfer functions

$$
G_{1}(s)=\frac{1}{2 s^{2}+6 s+3} e^{-T s}, \quad G_{2}(s)=\frac{5 s+1}{10 s^{2}+10 s+3} e^{-T s} .
$$

In equation (16) variable $T$ represents the time delay. The impulse response of the original system and the approximation with the GLFs and SLFs can be seen in Fig. 2. The legend shows the values of the free 


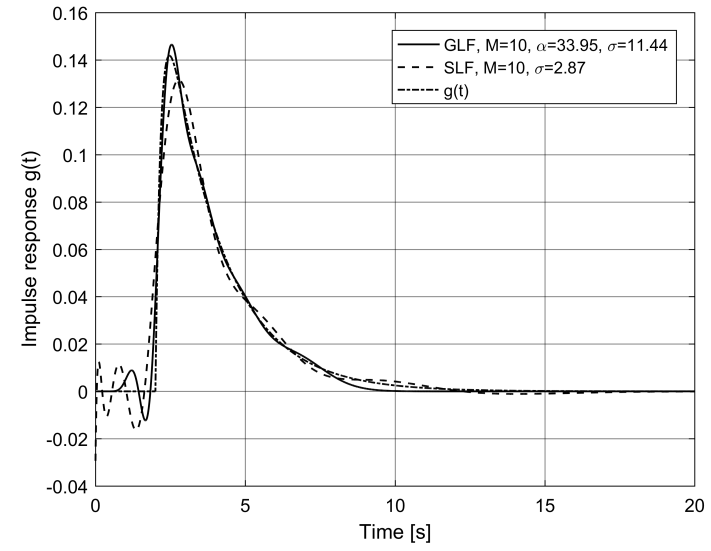

(a) Impulse response of system $G_{1}(s), T=2 s$

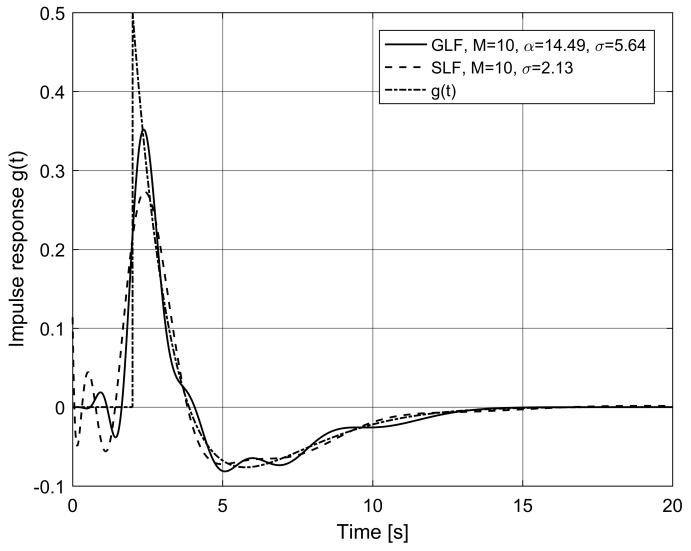

(b) Impulse response of system $G_{2}(s), T=2 s$

Figure 2: Impulse responses and their approximation with Laguerre functions

parameters used to obtain the results. It can be seen from both figures that for order $M=10$ the GLFs give better results.

Fig. 3 shows the values of the quadratic criterion for system $G_{1}(s)$ for different values of time delay. It can be seen that the error decreases rapidly with the increasing order of the approximation for both the GLFs and SLFs. For both values of time delay the GLFs give better results for all orders of approximation.

In Fig. 4 the quadratic errors are displayed for the second system $G_{2}(s)$ for different values of time delay. As one can see, Fig. 4a shows similar results to Fig. 3a. On the other hand, the quadratic errors in Fig. 4b are different from Fig. 3b. In Fig. 3b the errors obtained with the GLFs are close to zero even for low orders of approximation. In contrast, the SLFs give better results for some of the lower orders of approximation in Fig. 4b. Nonetheless, the GLFs still give smaller errors for the higher orders. This is in accordance with the conclusions drawn in [1]. The next chapter will explain why are the results different for the two systems for low orders of approximation with small time delay.

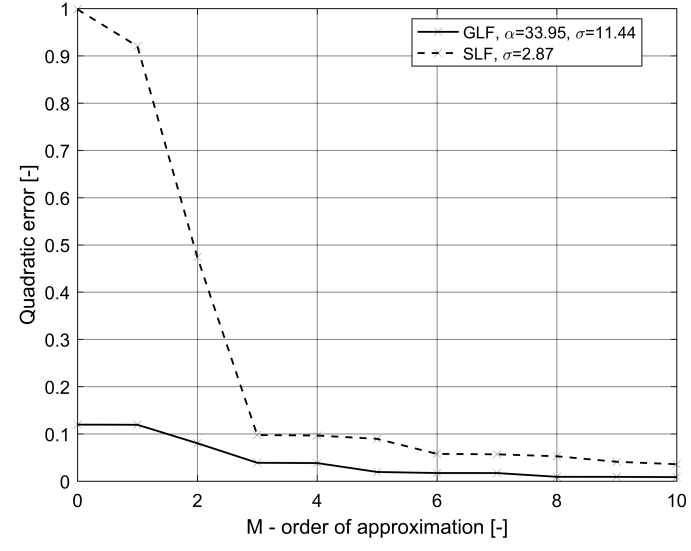

(a) System $G_{1}(s)$ with time delay $T=2 s$

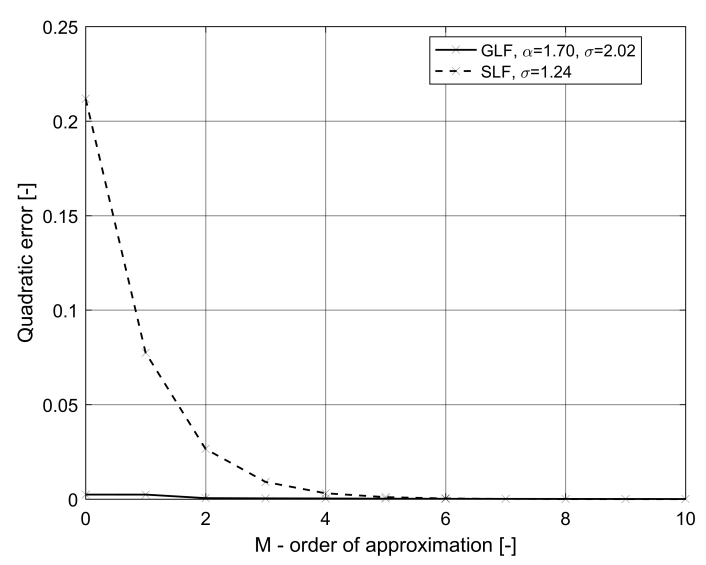

(b) System $G_{1}(s)$ with time delay $T=0.01 s$

Figure 3: Comparison of the quadratic errors for system $G_{1}(s)$

\subsection{Results for Low Order of Approximation}

As already discussed in [1], the approximations with the generalized Laguerre functions give better results. Exceptions are the low order of the approximation and small values of time delay. In this case for some systems the GLFs, for others the SLFs create more accurate approximations. Examples of this are displayed in Fig. 3b and Fig. 4b. The reason for this will be explained through the properties of the approximated systems.

The transfer function of linear time-invariant systems is given by

$$
G(s)=\frac{B_{p}(s)}{A_{r}(s)},
$$




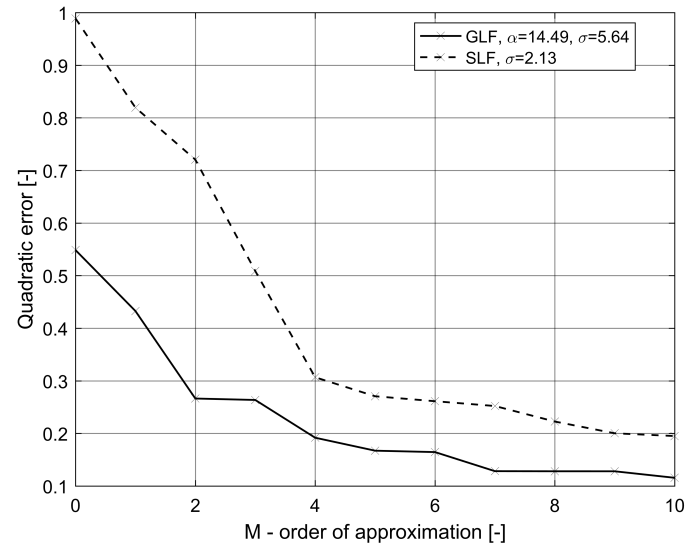

(a) System $G_{2}(s)$ with time delay $T=2 s$

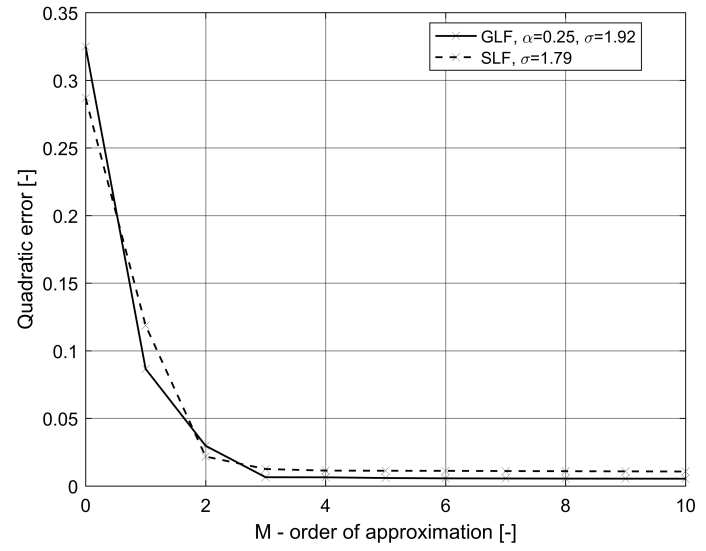

(b) System $G_{2}(s)$ with time delay $T=0.01 \mathrm{~s}$

Figure 4: Comparison of the quadratic errors for system $G_{2}(s)$

where $B_{p}(s)$ and $A_{r}(s)$ are polynomials with order $p$ and $r$ respectively. $G(s)$ is also the Laplace transform of the system's impulse response $g(t)$ :

$$
G(s)=\mathcal{L}\{g(t)\}
$$

According to the initial value theorem, the value of the impulse response for $t=0$ can be calculated as

$$
\lim _{t \rightarrow 0^{+}} g(t)=\lim _{s \rightarrow \infty} s G(s)=\lim _{s \rightarrow \infty} s \frac{B_{p}(s)}{A_{r}(s)}= \begin{cases}\frac{b_{p}}{a_{r}}, & r-p=1 \\ 0, & r-p>1 \\ \infty, & r-p<1\end{cases}
$$

There are no real systems which meet the requirement $r-p<1$, so this case will not be discussed. The other two cases are satisfied by systems $G_{1}(s)$ and $G_{2}(s)$ according to Table 1 .

Table 1: Values of the impulse response according to the initial value theorem

\begin{tabular}{lll}
\hline System's order & $g(0)$ & Example \\
\hline$r-p=1$ & $\frac{b_{p}}{a_{r}}$ & $G_{1}(s)$ \\
$r-p>1$ & 0 & $G_{2}(s)$ \\
\hline
\end{tabular}

Table 1 shows the initial values of the impulse responses for different systems. If the system contains time delay then the impulse response is shifted by $T$. This means that the values for $t=0$ are also shifted to $t=T$. This can be seen in Fig. 2. System $G_{1}(s)$ has a value of zero in $t=T$ and the value of system $G_{2}(s)$ jumps to 0.5 in $t=T$.

In section 3 it was stated that the SLFs' value in $t=0$ is always $\sqrt{\sigma}$ and the GLFs' value in this point is zero. This property is similar to the value of the impulse responses in $t=T$. This is the reason why the generalized functions give better results even for low orders and small time delays for systems where $r-p>1$. For systems where $r-p=1$ the errors obtained with the simple and generalized Laguerre functions are similar in value.

\section{Conclusion}

This paper explained the results of time-delay system approximations with the simple and generalized Laguerre functions for low orders of approximation. The results of the approximations were shown on two systems. The difference between the systems lies in the order of the transfer functions' polynomials. This has an impact on the initial value of the impulse responses. It was shown that this makes a difference in the results of the approximations for lower orders. The main reason for this is that the generalized and simple Laguerre functions 
have different values in $t=0$, similarly to the two systems. As it can be seen in Fig. $3 \mathrm{~b}$, the error obtained from the approximation with the generalized Laguerre functions is smaller. This is because in the case of system $G_{1}(s)$ the difference in the order of the transfer functions' polynomials is greater than one. For the other system, the simple Laguerre functions give better results for some of the lower orders of approximation. This can be seen in Fig. 4b. The reason for this is that the difference between the orders of the transfer functions' polynomials is one.

Acknowledgement: The completion of this paper was made possible by the grant No. FEKT-S-17-4234 - "Industry 4.0 in automation and cybernetics" financially supported by the Internal science fund of Brno University of Technology.

\section{References}

[1] Zsitva, N. 2019. Comparison of generalized and simple Laguerre functions for time-delay system approximation. In Proceedings of the 25th Conference STUDENT EEICT 2019, Brno University of Technology, Brno, pp. 581-585. ISBN: 978-80-214-5735-5

[2] Belt, H. J. W. and den Brinker, A. C. 1997. Optimal parametrization of truncated generalized Laguerre series. In 1997 IEEE International Conference on Acoustics, Speech, and Signal Processing. IEEE, Berlin, Germany. DOI: 10.1109/ICASSP.1997.604708

[3] Fischer, B. R. and Medvedev, A. 1999. $L^{2}$ Time Delay Estimation by Means of Laguerre Functions. In Proceedings of the 1999 American Control Conference. IEEE, San Diego, CA, USA, pp. 455-459. DOI: 10.1109 /acc.1999.782869

[4] Belt, H. J. W. and den Brinker A. C. 1998. Optimal free parameters in orthonormal aproximations. IEEE Transactions on Signal Processing 46, 8, pp. 2081-2087. DOI: 10.1109/78.705414

[5] Parks, T. W. 1971. Choice of time scale in Laguerre approximations using signal measurements. IEEE Transactions on Automatic Control 16, 5, pp. 511-513. DOI: 10.1109/TAC.1971.1099780 